\title{
Isolation of the Mutual Longitudinal Dipolar Relaxation of a Pair of Coupled Spins
}

\author{
amirah Chaudhry, José Pereira, * and Timothy J. Norwood \\ Department of Chemistry, University of Leicester. University Road. Leicester LEI 7RH, United Kingdom: \\ and * Instituto de Ciencias Biomedicas de Abel Salazar. Universidade do Porto, Portugal \\ Received September 9, 1994
}

The NMR relaxation properties of a coherence or spin order can potentially provide detailed and invaluable information on molecular dynamics. This information has been shown to be important in determining protein structure, internal dynamics, and interactions (1-4). Relaxation has been used to measure the amplitude of internal motions and their correlation times as well as the overall correlation time of a molecule $(5,6)$.

Unfortunately, except in special circumstances this potential usually goes unrealized because the relaxation processes that occur are often complex and consequently too difficult to analyze. For a feasible quantitative analysis the number of variables must be restricted. In this Communication we present a method that achieves this by enabling the mutual longitudinal dipolar relaxation of an arbitrary pair of scalar coupled spins to be isolated from all other interactions. We outline the basis of this method theoretically and discuss and demonstrate experimental procedures for making the required measurements.

There are a few cases where circumstances have allowed the mutual relaxation of a few spins to be considered to be in isolation from all others. For example, for relaxation of ${ }^{15} \mathrm{~N}$ and ${ }^{13} \mathrm{C}$ in ${ }^{15} \mathrm{~N}-\mathrm{H}(5,7)$ and ${ }^{13} \mathrm{CH}_{n}$ groups $(6,8,9)$ in a labeled protein, the assumption can be made that the heteronucleus relaxes solely through dipolar interactions with its bonded proton(s) and its own chemical-shift anisotropy (CSA). Distance makes all other dipolar interactions relatively weak due to the $\left(\gamma_{i}^{2} \gamma_{j}^{2} / r_{i j}^{6}\right)$ dependency of dipolar relaxation between a pair of spins $i$ and $j$. Both of these isotopes are naturally low in abundance $(<1 \%)$ and hence they also have very low NMR sensitivitics at the concentrations used in NMR experiments. Therefore to measure ${ }^{15} \mathrm{~N}$ or ${ }^{13} \mathrm{C}$ relaxation rates, it is usually necessary to label the sample. To make full use of the information implicit in NMR relaxation, we need to be able to measure the mutual relaxation of any arbitrary pair of nuclear spins. For this to be feasible it is necessary to find a way to isolate the relaxation of a pair of spins from all other interactions. To this end we consider the time dependence of the density operator, $\boldsymbol{\sigma}$, which can be described in matrix form by

$$
\frac{d \mathbf{\sigma}(t)}{d t}=-[i \mathbf{H}+\mathbf{\Gamma}]\left(\boldsymbol{\sigma}(t)-\mathbf{\sigma}_{0}\right),
$$

where $\mathbf{H}$ is the time-independent nuclear spin superoperator which contains information about the spin variables such as chemical-shift and scalar coupling constants and $\boldsymbol{\Gamma}$ is the relaxation superoperator. Since we are concerned here with longitudinal relaxation modes, $\mathbf{H}$ will not be considered any further. The density operator $\boldsymbol{\sigma}(t)$ is usually expanded as a series of operators corresponding to various types of longitudinal magnetization and spin order, $I_{i z}$ is the longitudinal magnetization of spin $i, 2 I_{i z} I_{j z}$ is the longitudinal two-spin order of spins $i$ and $j$, and $4 I_{i z} I_{j z} I_{k z}$ is the longitudinal threespin order of spins $i, j$, and $k$. At equilibrium all modes except longitudinal magnetization have zero intensity.

The relaxation Hamiltonian, $\mathscr{H}_{1}$, can be written as

$$
\mathscr{H}_{1}(t)=\sum_{q} F^{(q)}(t) A^{(q)},
$$

where the operators $A^{(q)}$ act on the variables of the system and $F^{(q)}(t)$ are random functions of time $(10,11)$. When the operators are expanded into eigenoperators, $A_{p}^{(q)}$, of the unperturbed Hamiltonian $(10,12)$, evolution resulting from relaxation can be written as

$$
\frac{d \boldsymbol{\sigma}(t)}{d l}=-\frac{1}{2} \sum_{p, q, q^{\prime}} J_{q q^{\prime}}\left(\omega_{p}^{(q)}\right)\left[A_{p}^{\left(-q^{\prime}\right)},\left[A_{p}^{(q)}, \boldsymbol{\sigma}(t)-\boldsymbol{\sigma}_{0}\right]\right]
$$

where the power spectral-density function is

$$
J_{q q^{\prime}}\left(\omega_{p}^{(q)}\right)=\int_{-\infty}^{\infty} \overline{F^{(q)}(0) F^{\left(q^{\prime}\right) *}(\tau)} \exp (-i \omega \tau) d \tau .
$$

Elements of the relaxation matrix can be readily calculated from Eq. [3]. Elements corresponding to the transverse re- 